\title{
A Case of Subacute Combined Degeneration as a Manifestation of Pernicious Anaemia
}

\author{
Authors: \\ * Marta Arriaga Rocha, Martim Trovão Bastos, Joana Mauríco, Susana \\ Heitor \\ Internal Medicine Service 3, Hospital Professor Doutor Fernando Fonseca, EPE, \\ Amadora, Portugal \\ *Correspondence to marta_arriaga_rocha@hotmail.com \\ Disclosure: $\quad$ The authors have declared no conflicts of interest. \\ Received: $\quad 03.03 .21$ \\ Accepted: $\quad 25.05 .21$ \\ Keywords: $\quad$ Anaemia, atrophic gastritis, intrinsic factor, myelopathy, vitamin $\mathrm{B}_{12}$. \\ Citation: $\quad$ EMJ Neurol. 2021;9[1]:77-80.
}

\begin{abstract}
Vitamin $B_{12}$ deficiency affects multiple systems, including the central and peripheral nervous systems, producing a vast spectrum of neurological symptoms. It is particularly important due to its insidious presentation and because it can evolve to spastic paraplegia with permanent sequelae. The authors describe a case of a woman with asthenia, bilateral lower limb weakness, urinary retention, and faecal incontinence, with no structural cause on imaging studies. Blood tests showed anaemia (haemoglobin: $6.8 \mathrm{~g} / \mathrm{dL}$ ) and vitamin $B_{12}$ deficiency ( $<100 \mathrm{pg} / \mathrm{mL}$ ). After upper digestive endoscopy compatible with chronic atrophic gastritis and positive for anti-intrinsic factor antibodies was obtained, the diagnosis of subacute combined degeneration due to vitamin $B_{12}$ deficiency in the context of pernicious anaemia was admitted. Although this entity is a rare cause of myelopathy, it is a frequent manifestation of vitamin $\mathrm{B}_{12}$ deficiency. Clinical suspicion is fundamental since the reversibility of the neurological lesion is dependent on early treatment.
\end{abstract}

\section{INTRODUCTION}

Vitamin $B_{12}$ (cobalamin) deficiency is a highly frequent condition that affects approximately $6 \%$ of the population in developed countries, particularly institutionalised or malnourished elderly people (20\% prevalence in $>60$ years old). ${ }^{2}$ Despite often being asymptomatic, this deficiency may impact several organs and systems, with gastrointestinal, haematological, cardiovascular, psychiatric, and neurological manifestations.

Vitamin $B_{12}$ deficiency is associated with a wide spectrum of neurological effects in central and

peripheral nervous systems. Subacute combined degeneration (SCD), the myelopathy caused by this deficiency, initially affects the dorsal and lateral columns of the spinal cord, but it can extend to pyramidal tracts and lead to spastic paraparesis. It is thus critical to rapidly diagnose and treat vitamin $B_{12}$ deficiency to prevent the development of serious and permanent neurological complications., ${ }^{1,2}$

Herein, the authors present a case of a woman with asthenia, lower limb paresis, urinary retention, and faecal incontinence, diagnosed with SCD due to vitamin $\mathrm{B}_{12}$ deficiency. 


\section{CASE REPORT}

A 65-year-old melanodermic woman from Guinea-Bissau, autonomous in everyday life and with a medical history of Type 2 diabetes mellitus treated with metformin, without any other medication or drug allergies, presented to the emergency department with asthenia and bilateral lower limb weakness with 10 days of evolution, and recent complaints of low back and hypogastric pain associated with dysuria and decreased urine output.

On admission, she was conscious, normotensive (128/86 $\mathrm{mmHg}$ ), normocardial (75 bpm), and apyretic (tympanic temperature: $36.8^{\circ} \mathrm{C}$ ). Heart and lung auscultation had no alterations. She had abdominal tenderness at hypogastrium palpation, with evidence of a mass that was suggestive of a bladder globe. She was catheterised, with urine drainage of $500 \mathrm{~mL}$, suggesting urinary retention.

Neurological examination revealed symmetric ataxia of lower limbs and mild hyporeflexia, but unimpaired segmental and global strength as well as muscle tone. No significant alterations in pain sensitivity or sensitive level were detected. Bilateral apallesthesia, multiple proprioceptive errors, and marked gait instability were observed. The patient was oriented in time and space, without cognitive or speech alterations, as confirmed by a relative.

She had macrocytic anaemia with haemoglobin: $6.8 \mathrm{~g} / \mathrm{dL}$; mean corpuscular volume: $98.3 \mathrm{fL}$ and normal numbers of other cells; creatinine: 1.21 $\mathrm{mg} / \mathrm{dL}$; urea: $43.6 \mathrm{mg} / \mathrm{mL}$; protein-to-creatinine ratio: $22.34 \mathrm{mg} / \mathrm{dL}$; and pyuria. No acute changes were observed in cranial CT, whereas lumbar spine CT revealed incipient degenerative skeletal changes with no apparent compromise at the root level or at the spinal channel. The patient was admitted to the hospital for further study of the neurological changes, treatment of serious anaemia, and likely urinary tract infection.

She was given a transfusion of one unit of packed red blood cells with good transfusional yield, and amoxicillin plus clavulanic acid for 7 days to treat the infection due to a multi-sensitive Escherichia coli. Lower limb and pelvic girdle paresis as well as ataxia worsened, with urinary retention episodes and faecal incontinence. There was no evidence of structural lesions as assessed by cranial and spine MRI. Lumbar puncture revealed low cellularity, mainly mononuclear cells, with normal proteinorrachia and glychorrachia. HIV and venereal disease research laboratory test serologies were negative. Vitamin $B_{12}$ levels were $<100 \mathrm{pg} / \mathrm{mL}$, suggesting a serious cobalamin deficiency.

Autoantibody serology was positive for antiintrinsic factor antibodies and negative for anti-parietal cell antibodies. No macroscopic changes were observed in upper gastrointestinal endoscopy, but the anatomo-pathological examination showed chronic gastritis of the antrum, body, and fundus, without activity and with mild atrophy. These findings supported a diagnosis of SCD and megaloblastic anaemia due to vitamin $B_{12}$ deficiency, in the context of pernicious anaemia.

Other vitamin deficiencies, particularly in group $B$, were not investigated since the patient did not have a clinical picture of malnutrition but rather a specific deficit in the absorption of vitamin $B_{12}$.

The patient was given daily intramuscular injections of $1 \mathrm{mg}$ cyanocobalamin for 7 days, followed by weekly injections for a month, combined with muscle strengthening training, which resulted in significant progressive improvement. She was discharged 42 days after admission, exhibiting clinical improvement with autonomous although ataxic gait and no evidence of faecal incontinence or urinary retention. Monthly treatment with $1 \mathrm{mg}$ cyanocobalamin and physiotherapy were prescribed. At re-evaluation 6 months later, she was asymptomatic, with no objective neurological deficits, haemoglobin $12.4 \mathrm{~g} / \mathrm{dL}$, and vitamin $B_{12}$ levels $>300 \mathrm{pg} / \mathrm{mL}$.

\section{DISCUSSION}

B vitamins are a group of water-soluble vitamins that have some general similarities but are each unique with critical functions in the body. Collectively called B complex vitamins, they are sorted into eight chemically distinct entities: thiamine $\left(B_{1}\right)$, riboflavin $\left(B_{2}\right)$, niacin $\left(B_{3}\right)$, pantothenic acid $\left(B_{5}\right)$, pyridoxine $\left(B_{6}\right)$, biotin $\left(B_{7}\right)$, folate $\left(B_{9}\right)$, and cobalamin $\left(B_{12}\right)$. They are all co-factors for enzymatic reactions, providing important catalytic functionality to drive metabolic processes to completion, and cannot be synthesised in the human body. 
Therefore, they must be consumed through food sources. ${ }^{3}$

Vitamin $B_{12}$ is found in a wide variety of animal foods and fortified plant foods. Its absorption depends on intrinsic factor, a protein produced by parietal cells of the gastric mucosa. It forms a complex with vitamin $B_{12}$ in the small intestine that is absorbed in the terminal ileum. ${ }^{1,2}$ The main causes of significant vitamin $\mathrm{B}_{12}$ deficiency are related to malabsorption, either due to intrinsic factor deficiency (pernicious anaemia) or due to gastric (total or partial gastrectomy) or ileal pathology (Crohn's disease, ileal resection). ${ }^{4}$

Vitamin $B_{12}$ is a co-factor required for the formation of tetrahydrofolate, a metabolite involved in purine and thymidine biosynthetic pathways, which are essential for DNA maturation in proliferating cells (excluding haematopoietic stem cells), as well as for conversion of homocysteine into methionine and for methylmalonic acid metabolism, both necessary to maintain neuronal homeostasis. Therefore, vitamin $B_{12}$ deficiency is often associated with megaloblastic anaemia, with possible involvement of other cell lineages and neurological symptoms. ${ }^{5}$

Vitamin $B_{12}$ deficiency is linked to progressive demyelination, the physiopathological mechanism of which remains poorly understood. It is thought that methylmalonic acid accumulation prevents normal myelin synthesis, leading to abnormal fatty acid accumulation in neuronal sheaths. Axonal loss due to vacuolar degeneration of white matter, as well as decreased synthesis of methionine-dependent neurotransmitters, might also be involved. The major affected areas include the spinal cord, brain white matter, peripheral nerves, and optic nerve. ${ }^{6,7}$

The myelopathy caused by vitamin $\mathrm{B}_{12}$ deficiency is characterised by the involvement of posterior and lateral columns of the cervical and thoracic spinal cord. Patients usually present with insidiously developing symptoms that start with paresthesia and progressively evolve into symmetrical sensory disturbances, areflexia, and ataxia. Lower limbs are typically affected before upper limbs, with gait impairment. In advanced stages of disease, paresis and stiffness may develop, as well as a dementia syndrome with possible psychotic symptoms. ${ }^{2,6,7}$ MRI findings of the posterior columns, showing areas of abnormally increased T2 signal hyperintensity associated with spongy degeneration of myelin fibres, have been documented. ${ }^{8}$

SCD diagnosis is based on laboratory-confirmed absolute or relative vitamin $B_{12}$ deficiency in people with suggestive neurological symptoms without any other cause. Anaemia is absent in $20 \%$ of the cases. ${ }^{9}$ Vitamin $B_{12}$ levels $<200 \mathrm{pg} / \mathrm{mL}$ are specific of vitamin $B_{12}$ deficiency and increase the probability of response to supplementation, although with a diagnostic sensitivity of $60-95 \% .^{3}$ In patients with a borderline value of vitamin $B_{12}$ (200-400 pg/mL) and/or suggestive symptoms, the quantification of methylmalonic acid is recommended, with levels $>400$ $\mathrm{nmol} / \mathrm{L}$ displaying a sensitivity of $98 \%$ for clinically significant vitamin $\mathrm{B}_{12}$ deficiency. ${ }^{10}$ Antiintrinsic factor antibodies, which are present in $50 \%$ of patients with pernicious anaemia, have a very high diagnostic specificity when quantified before vitamin $\mathrm{B}_{12}$ supplementation. ${ }^{3}$

$\mathrm{SCD}$ is treated with vitamin $\mathrm{B}_{12}$ supplementation, which can be administered by parenteral or oral routes. Oral administration is given to healthy adults with vitamin deficiency, whereas parenteral administration is mandatory in patients with a vitamin $B_{12}$ deficiency due to malabsorption or associated with severe symptoms, including neuropathy. Parenteral supplementation consists of intramuscular injections of $1 \mathrm{mg}$ cyanocobalamin, in which approximately $10 \%$ of the dose is absorbed. In patients with anaemia, standard therapy is three injections per week for 2 weeks. Patients with neurological disorders should be subjected to a more intensive regimen, with daily or alternate injections until symptoms improve or until 3 weeks, followed by weekly injections. A monthly maintenance dose is also recommended of $1 \mathrm{mg}$ in patients with continuous vitamin $\mathrm{B}_{12}$ absorption deficiency (excluding pernicious anaemia). ${ }^{11}$

In contrast with the rapid increase of reticulocyte counts after 1 week and megaloblastic anaemia resolution after 6-8 weeks, the improvement of neurological symptoms is slow, being more evident during the first 2 months after starting treatment and continuing for the following 6 months. The severity and duration of the neurological changes are related to the grade and speed of the patient's recovery. ${ }^{12}$ 


\section{CONCLUSION}

vitamin $B_{12}$ deficiency as the aetiological agent of a wide range of neurological symptoms, given

The case report presented here, which describes a that neurological lesion reversibility is directly rare but reversible cause of myelopathy, highlights linked to an early diagnosis and correction of the importance of early clinical suspicion of vitamin deficiency.

\section{References}

1. Hunt $\mathrm{A}$ et al. Vitamin B12 deficiency. BMJ. 2014;349:95226.

2. Stabler SP. Clinical practice. Vitamin B12 deficiency. N Engl J Med. 2013;368(2):149-60

3. Kennedy DO. B vitamins and the brain: mechanisms, dose and efficacy - a review. Nutrients. 2016;8(2):68.

4. Langan RC, Zawistoski KJ. Update on vitamin B12 deficiency. Am Fam Physician. 2011;83(12):1425-30.

5. Dali-Youcef N, Andrès E. An update on cobalamin deficiency in adults. QJM. 2009;102(1):17-28.
6. Senol MG et al. Reversible myelopathy with vitamin B12 deficiency. Singapore Med J. 2008;49(11):e330-2.

7. Healton EB et al. Neurologic aspects of cobalamin deficiency. Medicine (Baltimore). 1991;70(4):229-45.

8. Yamada $\mathrm{K}$ et al. A case of subacute combined degeneration: MRI findings. Neuroradiology. 1998;40(6):398-400.

9. Quadros EV. Advances in the understanding of cobalamin assimilation and metabolism. $\mathrm{Br} J$ Haematol. 2010;148(2):195-204.
10. Savage DG et al. Sensitivity of serum methylmalonic acid and total homocysteine determinations for diagnosing cobalamin and folate deficiencies. Am J Med. 1994;96(3):239-46.

11. Devalia $\vee$ et al. Guidelines for the diagnosis and treatment of cobalamin and folate disorders. Br J Haematol. 2014;166(4):496-513.

12. Carmel R. How I treat cobalamin (vitamin B12) deficiency. Blood. 2008;112(6):2214-21. 